\title{
Modular Plug-and-Play Power Resources for Energy-Aware Wireless Sensor Nodes
}

\author{
A. S. Weddell, N. J. Grabham, N. R. Harris, and N. M. White \\ Electronic Systems and Devices Group, School of Electronics and Computer Science, \\ University of Southampton, Southampton, SO17 1BJ, UK. \\ \{asw,njg,nrh,nmw\}@ecs.soton.ac.uk
}

\begin{abstract}
Wireless sensors are normally powered by nonrechargeable batteries, but these must be replaced when depleted. Recent developments in energy harvesting technology allow sensors to be powered by environmental energy where it is present, but the wide range of situations where sensors are deployed means that it is desirable for the energy components of a sensor node (i.e. batteries, supercapacitors, and power generation devices) to be selected and configured at the time of node deployment. Previous energy harvesting-powered systems have been designed for specific energy hardware and been difficult to adapt for different resources. Energy-awareness is useful for state-of-the-art network algorithms, but present systems do not provide a standardized or straightforward way for nodes to monitor and manage their energy hardware.
\end{abstract}

The developments reported in this paper deliver a reconfigurable energy subsystem for wireless autonomous sensors. The new system permits energy modules to be selected and fitted to the sensor node in-situ, in a plug-and-play manner, without the need for reprogramming or the modification of hardware. The node can monitor and intelligently manage its energy resources and assess its overall energy status by analyzing its level of stored energy and rate of power generation. These activities are facilitated by a proposed common hardware interface (which allows multiple energy modules to be connected) and an electronic datasheet structure for the energy modules. The system has been verified through the development and testing of a prototype wireless sensor node which operates from a mix of energy sources.

Keywords-energy harvesting; energy management; wireless sensor networks

\section{INTRODUCTION}

Wireless sensor nodes interface with their sensing hardware, interpret the sensed parameter, and transmit the data wirelessly (normally via radio frequency communications) to other nodes on the network or a central data 'sink'. By definition, wireless sensors must not rely on a wired power supply, and are most often powered by non-rechargeable batteries. Batteries are a popular solution for this application, as they are cheap and have a high energy density, but they provide a limited amount of energy and must be replaced when depleted. The constrained nature of energy supplies for wireless sensor nodes, along with recent developments in

The work reported on in this paper was undertaken as part of the Data Information Fusion Defence Technology Centre (DIF DTC) Phase II 'Adaptive Energy-Aware Sensor Networks' project, funded jointly by the UK Ministry of Defence and General Dynamics UK. This work was also supported in part by the Engineering and Physical Science Research Council (EPSRC) under grant number EP/D042917/1. technology, mean that energy harvesting (the generation of electrical energy from other forms of energy in the sensor's environment - such as light, vibration, or temperature difference) is becoming an attractive way of powering sensors for long-term deployments or in situations where changing a battery is expensive or impractical.

Modern wireless sensor nodes utilize low-power microcontrollers such as the MSP430 or 8051 . For example the CC2430 system-on-chip device (which is based on an extended 8051 microcontroller) from Texas Instruments typically draws below $1 \mu \mathrm{A}$ when asleep. The device incorporates an IEEE 802.15.4-compliant transceiver and, when it is active and transmitting, draws a maximum of $27 \mathrm{~mA}$. It is capable of operating at supply voltages between $2.0 \mathrm{~V}$ and $3.6 \mathrm{~V}$ [1]. These properties mean that it is feasible for the operation of the device to be sustained by the power obtained from energy harvesting devices. Examples of devices operating from harvested energy include Prometheus from the University of California, Berkeley, which operates from outdoor solar energy [2]; and the VIBES demonstrator developed by the University of Southampton, which harvests energy from the vibration of machinery [3].

Very few projects have incorporated multiple energy resources onto a single node. AmbiMax, developed by the University of California, Irvine, is a notable example which combines energy harvesting from wind and light, and stores it in supercapacitors and lithium rechargeable batteries [4]. An advantage of the AmbiMax power module is that it is entirely analogue and autonomous. However, the system design must be adapted to accommodate changes of energy resource. Furthermore, the sensor node powered by the module has no means of finding out the levels of production or availability of energy as the output voltage of the module is fixed at $4.1 \mathrm{~V}$. An alternative system is MPWiNodeX, which is capable of using up to three energy sources to recharge a NiMH battery pack [5]. However, the type of energy store cannot be changed, and the energy sources only give a coarse indication of their status (they cannot be actively managed).

Energy-awareness is useful for state-of-the-art network algorithms, such as IDEALS/RMR [6], which aim to conserve energy when it is scarce and exploit it when it is plentiful. This is achieved by determining the importance of messages and controlling their reporting based on the energy status of the node. In this way, the node's operating time can be extended and the probability that the most important messages will be 
able to be transmitted is increased. However, delivering an accurate estimate of the energy status of a sensor node is a nontrivial task [7], and requires the node to have detailed knowledge of its hardware subsystem, which can be complex.

A family of IEEE standards, introduced in Section II, deliver plug-and-play capabilities to industrial transducers. In a similar way to how sensing transducer hardware can be reconfigured dependent on the requirement for measurements, it is desirable for the energy hardware of harvesting sensor nodes to be reconfigurable dependent on the power requirements of the node and the forms of energy available in the deployment environment.

This paper proposes a system which allows the energy resources on a sensor node to be connected and configured, at the time of system deployment, without the need to re-program the microcontroller software. The energy subsystem of the sensor node is split into a number of modules including the multiplexer (which facilitates the scheme). A common hardware interface is introduced, which permits the sensor node to communicate with each module individually and a preliminary Energy Electronic Datasheet (EEDS) format has been developed which stores module parameters in memories on the modules. The scheme as a whole allows the energy hardware of the sensor node to be configured and re-configured in-situ, with the sensor node being able to interrogate the electronic datasheet on each module to determine its operational parameters and how to interpret the obtained data.

A prototype system has been developed, which facilitates the flexible connection of devices as shown in Fig. 1. The scheme allows the sensor node to be powered by a selection of energy resources such as vibration and photovoltaic energy harvesting, primary batteries, and a mains power adapter. Energy may also be buffered in rechargeable batteries or supercapacitors. The system will autonomously start-up and detect changes in its energy subsystem, and accommodates up to six energy modules.

The developed scheme allows systems to be configured at the time of deployment, gives a great deal of flexibility with regard to the energy hardware, and allows the node to have a more detailed knowledge of its energy subsystem than has previously been possible. The system allows energy modules to be connected to any socket on the multiplexer module, and if necessary multiple modules of the same type can be connected.

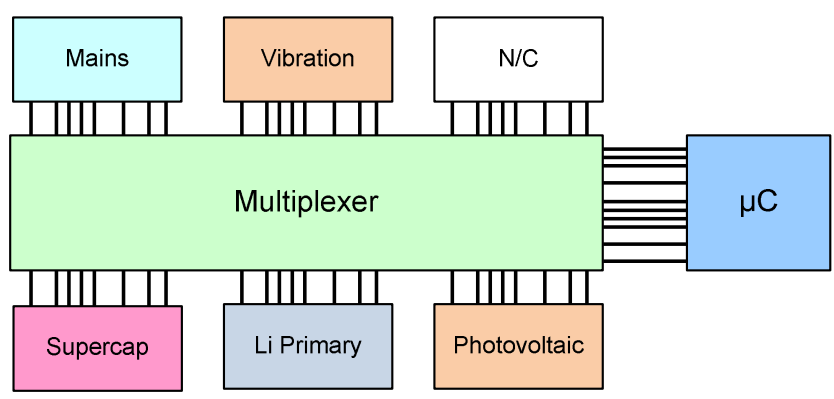

Figure 1. Example of the connections facilitated by the proposed scheme.
A major advantage of the plug-and-play functionality is that the system can be installed and configured by a person without an in-depth understanding of the operation of the sensor node. The complete system, with electronic datasheets and a common hardware interface, delivers a novel plug-and-play capability for the energy subsystems of wireless sensor nodes. The interface allows up to six energy modules to be used to power the sensor node, and the voltages and type of interface used means that the system can be utilized to power sensor nodes based on a range of low-power microcontrollers.

Section II introduces the concept and format of the EEDS, and Section III defines the common hardware interface which permits the energy subsystem to be interfaced with using one 8-bit microcontroller port. The module prototypes are described in Section IV, and the operation of the complete system is described in Section V. The performance of the system is evaluated in Section VI. The paper concludes in Section VII, which also discusses the planned future developments for the system.

\section{THE ENERGY ELECTRONIC DATASHEET}

\section{A. Background to electronic datasheets}

The IEEE 1451 standards family defines interfaces between industrial transducers and communication networks, with the aim of delivering plug-and-play capabilities to industrial sensor systems. The standards define common interfaces, management structures, and electronic datasheet templates for transducers. The most relevant standard to this paper is IEEE 1451.4-2004, which defines a mixed-mode (analogue and digital) interface between transducers and application processors, along with the format of the Transducer Electronic Datasheet (TEDS) [8]. Systems that are compliant with this standard allow transducers to be installed without the need for manual configuration. A 1451.4-compliant system will normally consist of a smart transducer that is connected to a data acquisition system which interfaces through a network-capable application processor to a display device such as a PC.

An example of a typical smart sensor is from the Watlow INFOSENSE-P transducer family, which implements the IEEE 1451.4 standard. As shown in Fig. 2, the smart sensor has its TEDS calibration data stored in an EPROM on the device, rather than the conventional approach of printing the information on a tag attached to the sensor. The process of installing, identifying, and calibrating the transducer is therefore automated and takes place as soon as the device is attached to the system.

\section{B. Extensions to the scheme}

The concept of electronic datasheets has been taken further by Bandari et al. [10], who have developed the Component Electronic Datasheet which stores information about electrical and mechanical components in a system. This forms part of a process for Integrated Systems Health Management (ISHM) in which overall reliability is optimized by increasing the level of information stored about each individual component's operating parameters, in the case of their example for a rocket testing facility. 


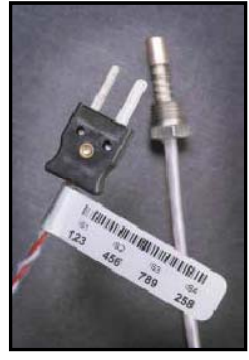

(a)

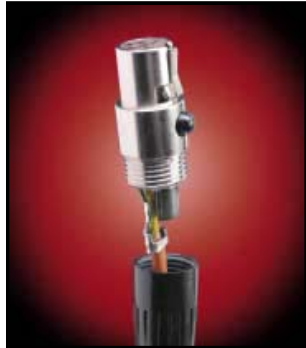

(b)
Figure 2. (a) A conventional transducer with tag giving calibration information which must be entered manually or using the barcode stripe, and (b) A 1451.4-compliant transducer which incorporates calibration information on its EPROM. Reproduced from Watlow datasheets [9]

In this paper, we propose using an Energy Electronic Datasheet (EEDS) to store parameters of energy modules. The aim of the EEDS is to promote the reconfigurability of the energy resources of sensor nodes by storing parameters related to the operation of each energy module on the modules themselves. The datasheet identifies the type of module (e.g. photovoltaic energy harvester) and its operating parameters (e.g. how the power generated corresponds to its open-circuit voltage and which data lines to use to query the device). This enables autonomous sensors to monitor and manage the status of their energy modules with greater reliability and accuracy in order to more effectively deliver energy-aware operation.

\section{Hardware and interrogation method}

TEDS is most commonly implemented on Maxim-Dallas 1wire EPROMs [11]. 1-wire devices communicate using a timing-specific protocol and require only two connections: ground and a combined data and power pin. The capabilities of the 1-wire protocol mean that it is possible for a large number of devices to share the same single-wire bus. However, the addressing of these devices in this situation is not straightforward as the master has to go through an initial search process to identify all devices on the line in order to determine their serial numbers. When it has done this, it will still be uncertain about the actual physical location of each device. Further detail on the hardware interface implemented in this system is given in the following section.

The EEDS implemented in this system uses the DS2502+ $1 \mathrm{kB}$ add-only EPROM. Each device has a unique serial number and 1024 bits of programmable memory, partitioned into four pages. The device is rated for operation between $2.8 \mathrm{~V}$ and $6.0 \mathrm{~V}$ from $-40^{\circ} \mathrm{C}$ to $+85^{\circ} \mathrm{C}$. Its operation in this system, however, has been successfully tested down to $2.0 \mathrm{~V}$ at room temperature. The device must be programmed before deployment as it requires programming voltages of up to $+12.0 \mathrm{~V}$, which cannot be realized by the sensor node.

\section{Outline datasheet structure}

The basic EEDS structure implemented in this demonstrator is as shown in Table I. This permits the microcontroller to obtain sufficient information to query, interpret and manage the energy modules. It is expected that, for later revisions of the datasheet format, descriptors such as manufacturer ID and model number will also be included. Full details on the codes used for each field cannot be included in
TABLE I. PRELIMINARY EEDS FORMAT

\begin{tabular}{|c|c|c|}
\hline Field & Size & Description \\
\hline Serial Number & 64 bits & Device ID of EPROM \\
\hline Module Type & 8 bits & Identifies class of module \\
\hline Bidir Direction & 8 bits & I/O direction of bidir pins \\
\hline Bidir 1 Function & 16 bits & \multirow{4}{*}{$\begin{array}{l}\text { Function of bidirectional lines - } \\
\text { how to query and interpret } \\
\text { results }\end{array}$} \\
\hline Bidir 2 Function & 16 bits & \\
\hline Bidir 3 Function & 16 bits & \\
\hline Bidir 4 Function & 16 bits & \\
\hline Parameters & Variable & Dependent on module type \\
\hline
\end{tabular}

this paper due to space restrictions, and are currently subject to change as the system is further developed.

\section{COMMON HARDWARE INTERFACE}

\section{A. Requirements}

Microcontrollers have a limited number of $\mathrm{I} / \mathrm{O}$ pins available, especially those that are connected to the ADC, and it is necessary to conserve these as far as possible. Furthermore, within our scheme of EEDS-equipped modules, it is important that the microcontroller is able to access each module independently - firstly in order to determine its datasheet parameters, and later to perform measurement and control operations on each module. In the scenario outlined earlier, it would be unrealistic to expect that each module would have multiple direct connections to $\mathrm{I} / \mathrm{O}$ pins of the microprocessor. For this reason, it is necessary to multiplex parts of the system to make best use of resources and allow queries to be effectively executed.

A drawback of 1-wire devices is that they draw current when connected even when not actively communicating. In order to isolate all 1-wire devices, the first multiplexed address (address 0 in this case) is used for ancillary communication between the microcontroller and the multiplexer, without a 1wire device. The microcontroller leaves the address lines in this state when not actively communicating with the energy modules. This permits the multiplexer module to use the bidirectional data lines to trigger interrupts on the microcontroller (e.g. they can be used to indicate that a voltage threshold has been reached). The allocation of two addresses to the multiplexer module leaves six further addresses for other energy modules.

\section{B. Structure}

With the conventional method of searching a 1-wire bus outlined earlier, in this application it would still be unclear which module is connected to each multiplexer module socket (hence making monitoring or control of energy modules through their correct sockets impossible). In this system, the 1wire devices are connected via the multiplexer module in order that they can be accessed one module at a time (by socket number rather than serial number), and the multiplexer module's EPROM is always accessed through the final address (address 7). The scheme shown in Table II is used to define the multiplexed address of each module (with this number of devices, there needs to be three multiplex address lines).

By first querying the final address (the multiplexer module) the EEDS of this module can be read in order to determine its operating parameters. These parameters include the minimum 
cut-off voltage, maximum supply rail voltage, and voltage regulation scheme. In this way, and through this common interface, the microcontroller can self-determine the energy subsystem's parameters through the sequential querying of the EEDS on all devices attached through the multiplexer module. It will also understand the function of each bi-directional line. In order to provide the maximum level of flexibility, the lines listed in Table III are provided between the microcontroller and the multiplexer module (two lines are the power supply to the microcontroller and the remaining eight are I/O lines, four of which must be equipped with ADCs).

It is the task of the multiplexer module to ensure that the supply voltage and ADC inputs that the microcontroller receives are not damaging to the device, nominally by means of diode-clamping to the supply output rails. The modules are also required to ensure that the measurement outputs they give are within the valid range, typically between $0 \mathrm{~V}$ and $1 \mathrm{~V}$ for low-voltage microcontrollers.

The interface between the multiplexer module and the energy modules is comprised of eight lines, as shown in Table IV. The 1-wire bus line and four bi-directional lines are passed through to the module after multiplexing. The ground and supply outputs from the module directly connect to those lines on the multiplexer module. Furthermore, the regulated voltage from the multiplexer module is fed back to each energy module

TABLE II. IDENTIFIERS FOR MODULES IN THE ENERGY SUBSYSTEM

\begin{tabular}{|c|l|l|l|}
\hline ID & Mux & Module Type & Example \\
\hline 0 & 000 & Multiplexer & Low-Power Monitoring \\
\hline 1 & 001 & Energy module & Supercapacitor \\
\hline 2 & 010 & Energy module & Lithium primary battery \\
\hline 3 & 011 & Energy module & Photovoltaic harvester \\
\hline 4 & 100 & Energy module & DC from mains adapter \\
\hline 5 & 101 & Energy module & Vibration harvester \\
\hline 6 & 110 & Energy module & Not Connected \\
\hline 7 & 111 & Multiplexer & Active Interrogation \\
\hline
\end{tabular}

TABLE III. CONNECTIONS BETWEEN MICROCONTROLLER AND MULTIPLEXER MODULE

\begin{tabular}{|c|l|l|}
\hline Pin & Connector & Description \\
\hline 1 & Mux Address 0 & \\
2 & Mux Address 1 & Digital lines to control all multiplexers \\
3 & Mux Address 2 & \\
\hline 4 & 1-wire EEDS line & Digital 1-wire interface \\
\hline 5 & Bi-dir Recon A & \\
6 & Bi-dir Recon B & Lines to reconfigurable I/O pins on $\mu \mathrm{C}$ \\
7 & Bi-dir Recon C & (capable of both digital I/O and ADC) \\
8 & Bi-dir Recon D & \\
\hline 9 & Vreg & Supply voltage output (direct to node) \\
10 & GND & \\
\hline
\end{tabular}

TABLE IV. CONNECTIONS BETWEEN MULTIPLEXER MODULE AND ENERGY MODULES

\begin{tabular}{|c|l|l|}
\hline Pin & Connector & Description \\
\hline 1 & 1-wire EEDS line & Digital 1-wire EEDS interface \\
\hline 2 & Bi-dir Recon A & \\
3 & Bi-dir Recon B & Lines to reconfigurable I/O pins on $\mu \mathrm{C}$ \\
4 & Bi-dir Recon C & Directed via multiplexers \\
5 & Bi-dir Recon D & \\
\hline 6 & Vreg & Regulated voltage used as supply to $\mu \mathrm{C}$ \\
\hline 7 & Vout & Voltage interface \\
8 & GND & \\
\hline
\end{tabular}

to facilitate digital communications directly with the microcontroller without the need for level-shifting, otherwise the microcontroller could be exposed to out-of-range and damaging digital voltages.

In the scheme proposed here, it is not necessary to mandate specific sockets on the multiplexer module socket as all switching hardware is located on the modules themselves, rather than on the multiplexer module. However, it should be noted that each module is responsible for self-regulating its output voltage to a maximum of $4.5 \mathrm{~V}$. In the case of harvesting modules, this means that a voltage regulator, or voltagedetector and isolation transistor arrangement, will often be necessary.

\section{Connector selection}

As defined in tables III and IV, the interface between the multiplexer module and the energy modules requires 8 lines, and the interface between the microcontroller and the multiplexer has 10 lines. The cost of connectors is important, as is their robustness. It is expected that the energy modules will be connected and disconnected more frequently than connectors between the microcontroller and the multiplexer module. It is for this reason that RJ45 connectors are used for the interface between multiplexer and energy modules. The connection between the microcontroller and the multiplexer module is via a 2 x 5 -way 0.1 " pin header.

\section{Operation}

On initial system start-up, the EEDS on the multiplexer module is queried by the microprocessor on the sensor node. The microprocessor then interrogates the other modules in the energy subsystem. The important parameters are stored in the microcontroller's memory, and hence it is not necessary for the microcontroller to regularly query the EEDS on the modules as a matter of course.

In normal operation (after the configuration phase), the microcontroller periodically monitors the energy modules in order to gauge the energy status of the sensor node. To do this, the multiplex address lines are set to the device of interest, and the bi-directional reconfigurable lines are used to control transistors and obtain readings. For example, with the photovoltaic module, one of the lines is used as an output to control a transistor to disconnect the cell from its load and another line is used as an analogue input to the microcontroller through which the open circuit voltage of the cell can be measured. In other situations, the lines are used to connect the harvesting source to a known load to estimate the power being generated.

Some modules can be actively managed by the microcontroller. For example, it may be desirable to only switch a primary battery into the system if there is a highpriority message to be transmitted by the device and its energy status is low. In this situation, one of the bi-directional lines can be used to control the connection of the battery to the load (in normal operation, other lines can be used to gauge the stateof-charge of the battery or its connection status). Clearly on many modules the control line will need to have persistence. This is because the bi-directional lines are multiplexed and it is desirable for the microcontroller to be able to alter settings on a 
module and ensure that they will be maintained in their desired state after the multiplexer is switched away from their address. A bistable multivibrator will normally be used to achieve this.

Some modules, such as rechargeable batteries, require different types of control or more than one parameter to be measured, which is why four bidirectional reconfigurable lines have been allocated for these operations.

\section{E. Module requirements}

\section{Multiplexer module}

The purpose of the multiplexer module is to permit the interconnection of energy modules, to supply a stable power supply to the microcontroller, and to allow it to monitor and manage the energy modules through their data lines. The multiplexer module, as a minimum, features the following hardware:

1. 5x 8-to-1 multiplexers (4 bi-directional lines, 1 -wire line)

2. Undervoltage protection circuit to inhibit supply to $\mu \mathrm{C}$

3. Diode clamping on bi-directional lines

4. Pull-up resistor on $\mu \mathrm{C}$ side of 1-wire multiplexer

5. Pull-down resistors on address lines of multiplexers

6. Voltage regulation to $3.0 \mathrm{~V}$ for $\mu \mathrm{C}$ and 1 -wire devices

7. Query for internal voltage - scaled to $0-1 \mathrm{~V}$ range

8. Small low-ESR capacitor to act as operating buffer

9. 1-wire device programmed with EEDS

10. Up to six RJ45 sockets

The components selected for this module draw as little current as possible and do not adversely impact on the overall efficiency of the system. The multiplexers are low resistance and capable of operating at voltages down to $2.0 \mathrm{~V}$. The undervoltage protection circuit disconnects the microcontroller if the unregulated voltage of the system drops below $2.0 \mathrm{~V}$. The diode clamping on the bi-directional lines ensures that signals from the energy modules do not exceed the supply voltage of the microcontroller.

The 1-wire pull-up resistor facilitates 1-wire communications, and the pull-down resistors ensure that the multiplexers default to address 0 (for low-power monitoring). This also has the effect that the 1-wire pull-up resistor is isolated when the device is not active, thus the power consumption of the module is minimized.

Given that MSP430 microcontrollers have a typical supply voltage range of 1.8-3.6V [12], and the CC2430 requires 2.0$3.6 \mathrm{~V}$ [1], in order to support a wide range of microcontrollers, the multiplexer module regulates the supply voltage between $2.0 \mathrm{~V}$ and $3.0 \mathrm{~V}$. The multiplexer module supports an unregulated voltage that is higher than the maximum supply voltage of the microcontroller, and this will normally be $4.5 \mathrm{~V}$ as this is the maximum voltage supported by commercial supercapacitors.

\section{Other energy modules}

Each energy module will feature the following hardware:

1. Switches and diodes to prevent unwanted backflow of energy to harvesting devices from multiplexer module
2. Optional switching hardware to allow querying of energy status of module

3. Optional resistor divider to bring measurement in range of $\mu \mathrm{C}$ ADC, plus op-amp buffer

4. Optional persistent control channels

5. 1-wire device programmed with EEDS

6. Overvoltage protection limiting output to $4.5 \mathrm{~V}$, if necessary for the module

7. One RJ45 socket to connect with the multiplexer module

Essentially the purpose of energy modules is to ensure that they generate, buffer, or otherwise supply energy to the multiplexer module in order that it can reliably and efficiently supply power to the microcontroller. Given that the multiplexer module will operate with an unregulated voltage of up to $4.5 \mathrm{~V}$, it is expected that energy modules will self-regulate their outputs to a maximum of $4.5 \mathrm{~V}$. Each module is effectively able to operate autonomously, and must function in a stable manner without external control.

Care must be taken when designing energy modules to ensure that they will allow the system to start-up from cold. For example, if a system only has one energy harvesting module supplying power, it would be impossible for the system to start up if this module initializes as being disconnected or opencircuited. A further detail is that the energy module should not be damaged by not being connected to the multiplexer module i.e. they should not rely on their outputs being connected in order to maintain safe operation. As a general rule:

- $\quad$ Energy harvesters and mains modules should be designed to start up supplying energy to the system

- Fast-response buffers, such as supercapacitors, should default to allowing charge and discharge

- Slow-response buffers, such as rechargeable batteries, should be permitted to discharge (but may support a manual override facility, e.g. using push buttons), and automatically recharge if their output voltage is $<2.0 \mathrm{~V}$

- Primary energy sources (such as non-rechargeable batteries) will normally have a push-button control to allow the system to cold-start on initial installation, then be managed by the microcontroller

The following section describes the functionality of modules implemented in the demonstration system.

\section{Module Prototypes}

\section{A. Multiplexer module}

The multiplexer module, pictured in Fig. 3, supports the connection of up to six energy modules through its RJ45 sockets. It includes five Analog Devices ADG708 8-to-1 analogue switches. These operate from $1.8-5.5 \mathrm{~V}$, have a typical $3 \Omega$ on-resistance, and an enable pin. The maximum unregulated voltage supported by this module is $4.5 \mathrm{~V}$, and this is regulated down to $3.0 \mathrm{~V}$ by a Maxim XC6215 LDO linear regulator. A linear regulator was chosen for this design due to its simplicity and low quiescent power consumption. It supports currents of up to $150 \mathrm{~mA}$. Undervoltage protection is provided by a voltage detector and MOSFET combination, with the voltage detector being a 2.0V Maxim XC61C, having 


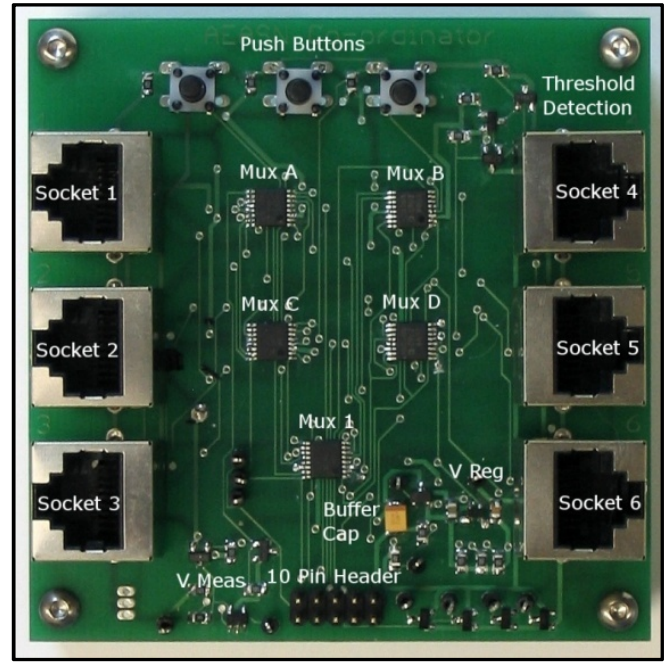

Figure 3. Multiplexer module.

a CMOS output stage. A tantalum $100 \mu \mathrm{F}$ capacitor provides a small low-ESR buffer which compensates for the current drain when the module output is turned on and the microcontroller starts up.

Additional circuitry includes pull-down resistors for the analogue switch address lines, and a pull-up resistor for the 1wire bus. Diode clamping is provided by an array of NXP BAT754 Schottky diodes. Three push switches are located on the top edge of the board. They are connected to the low-power monitoring lines of the system (address 0), permitting the triggering of interrupts to adjust parameters or trigger actions. Additionally, the facility is provided using an XC61C voltage detector and MOSFET arrangement to trigger an interrupt when the unregulated voltage passes $2.7 \mathrm{~V}$. This is an arbitrary selection but is used to show the ability of the system to indicate when a voltage threshold has been passed.

In order that the microcontroller can monitor the unregulated voltage on the multiplexer module, a resistor divider and op-amp (ST TS941) buffer arrangement is provided. This brings the potential $0-4.5 \mathrm{~V}$ unregulated voltage range into the $0-1 \mathrm{~V}$ range supported by most microcontroller ADCs. This facility is managed and monitored through address 7, and allows the microcontroller to monitor the unregulated voltage on demand.

\section{B. Photovoltaic module}

The photovoltaic energy harvesting module PCB is a development of the circuit reported in [13]. The photovoltaic cell and this PCB should be taken together to represent the photovoltaic module. The DS2502+ EPROM holds operating parameters of the circuitry and cell in combination, so it should be noted that in this scheme the actual harvesting device and its energy conditioning circuit are considered as one module.

The circuit interfaces with indoor amorphous silicon photovoltaic cells, preferably with an operating voltage of between $2.5 \mathrm{~V}$ and $5.0 \mathrm{~V}$. In the demonstrator system it is connected to a Schott OEM 1116929. This cell is capable of producing $>1 \mathrm{~mW}$ of power under bright indoor lighting. The circuit works to hold the cell at its optimal voltage, regardless of the unregulated voltage on the multiplexer module. This improves the efficiency of the system and delivers faster charging times when cold-starting the system.

Details on all components used for this module are beyond the scope of this paper, but it should be noted that the operating voltage of the photovoltaic module is set using a variable resistor. Overvoltage protection is delivered using a XC61C voltage detector and FET combination, which disconnects the photovoltaic cell when the maximum system voltage is reached. The same FET combination (feeding into an op-amp buffer arrangement) is used to permit the microcontroller to analyze the open-circuit voltage of the cell and hence estimate the nominal power being harvested.

\section{Vibration module}

The vibration module PCB is designed to interface with a Perpetuum PMG17 vibration energy harvester. The manufacturers have found, in real deployments, that this device can generate $500 \mu \mathrm{W}$ on $80 \%$ of machines, and $1.0 \mathrm{~mW}$ on $60 \%$ of machines [14]. It provides a half-wave rectified output limited to $8 \mathrm{~V}$. The circuitry on this PCB uses a high-efficiency step-down converter to limit this voltage to $4.5 \mathrm{~V}$. To increase the efficiency of the system, the device waits until the output capacitor is charged up before allowing energy to be output to the multiplexer module.

The nominal output power from the vibration energy harvester can be estimated by the microcontroller by shutting down the step-down converter and isolating its associated input capacitance. A known load is then switched in across the output of the vibration generator and the voltage across this load is measured to determine the instantaneous power level. The DS2502+ stores the characteristics of the energy harvester and the details of the known load.

\section{Supercapacitor module}

The supercapacitor module is designed to accommodate both CAP-XX thin, flat supercapacitors such as the GS206F $0.55 \mathrm{~F}$ model fitted in this prototype, and Panasonic Gold HW series supercapacitors. The DS2502+ EPROM stores information on the size and type of supercapacitor fitted.

Additional circuitry can be added to allow the microcontroller to query the stored voltage on the supercapacitor, but this has not been fitted as, in the configuration used in the demonstration, the voltage on the supercapacitor is the same as the unregulated voltage on the multiplexer module.

\section{E. Battery module}

The battery module is designed to accommodate a range of battery types. In the first prototype it is fitted with a $1 / 2 \mathrm{AA}$ size axial leaded lithium $3.0 \mathrm{~V}$ battery. It is also designed to accommodate a pair of AAA rechargeable NiMH batteries (in holders), or a CR2430 button cell. The DS2502+ EPROM stores information on the type and size of cell(s) fitted. The circuit is largely unpopulated in this instance as the battery fitted is a primary (non-rechargeable) cell. The additional circuitry permits the microcontroller to control the charging of rechargeable batteries fitted to the module.

There is currently no step-up capability, meaning that the module cannot supply a higher voltage than the voltage of the 
cell(s). It is intended that the next revision of this circuit will provide the capability to step the voltage up to $4.5 \mathrm{~V}$.

Circuitry is provided to allow the microcontroller to assess the state-of-charge of the battery. This is either by analyzing the open-circuit voltage, or the voltage after pulsed discharge through a small resistive load (set by resistor values soldered onto the board).

The board has two bistable multivibrators, which maintain the "discharge enable" and "charge enable" status of the module, meaning that these parameters can be asserted or negated by the microcontroller and that state will be maintained by the module. The two push-buttons on the bottom of the board are manual 'on' and 'off' switches, which permit the output to be manually enabled to allow the system to be coldstarted. Once the system has started, these can then be controlled by the microcontroller.

\section{F. Mains module}

The mains module permits the system to run from mains power. The PCB has a standard $2.1 \mathrm{~mm}$ jack socket and hence is compatible with a range of mains power adapters. The PCB will regulate a 5.0 to $11.5 \mathrm{~V} \mathrm{DC}$ input down to $4.5 \mathrm{~V}$, using a Maxim MAX639 switching regulator. The DS2502+ identifies the type of module and its pin functions. Additional circuitry permits the microcontroller to ascertain whether the mains adapter is supplying power to the system.

\section{SYSTEM INTEGRATION}

\section{A. Complete system}

A demonstration system, including a multiplexer module and four energy modules (supercapacitor, battery, photovoltaic and mains), is shown in Fig. 4. A further two energy module sockets on the multiplexer module are currently free. Energy modules can be connected to any RJ45 socket on the multiplexer module, and are connected here by standard $300 \mathrm{~mm}$ RJ45 patch leads. The energy subsystem shown is connected to Port 0 of a TI CC2430 evaluation module (EM) via a 10-way IDC cable. The interface with the CC2430 EM is via its battery board (without batteries attached).

\section{B. Default operation}

The default behavior of the system on first installation is to allow the energy harvesting device(s) to charge up the supercapacitor module. Once this store reaches approximately $2.1 \mathrm{~V}$, the system connects the power supply to the CC2430, which then starts up and tests its voltage. When this has reached a suitable level (nominally $2.7 \mathrm{~V}$ ) the microcontroller then performs the first energy-intensive tasks such as scanning its energy subsystem.

To deliver a near-instant start-up to the system, the 'On' button may be pressed on the primary battery module, which will cause the system to receive power from the battery. Once the microcontroller has taken control of the energy subsystem, the microcontroller disconnects the primary battery in order to conserve the charge level on the cell.

Alternatively, the mains adapter may be turned on, which would also act to rapidly charge up the supercapacitor module. However, the microcontroller cannot act to turn off this supply

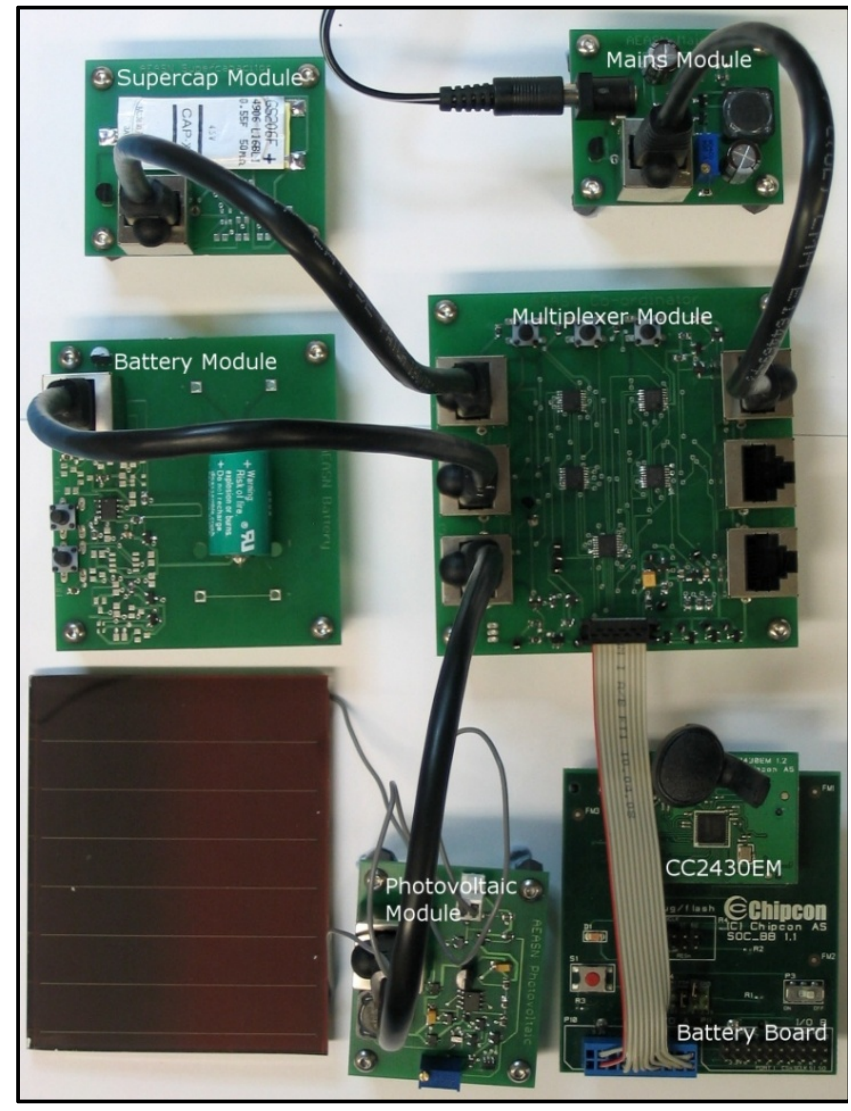

Figure 4. Complete system connection.

as it is assumed to be a zero-cost resource which should be taken advantage of whenever it is available.

The first scan of the energy subsystem is used to ascertain which sockets are occupied, what types of device are present, and their operating parameters. From this initial scan the microcontroller can reach an estimate of the amount of energy stored by the system. This data is stored in the microcontroller memory, so the EEDS of each module need only be scanned once (1-wire activities are power-intensive so it is undesirable to carry out unnecessary communications).

\section{Change detection}

The microcontroller keeps a table of which sockets on the multiplexer module are occupied. The microcontroller will periodically (at least once per minute when active) re-scan the sockets on the multiplexer, issuing a reset pulse on the 1-wire bus for each socket, then listening for a presence pulse in response. By comparing the presence pulses received against its table of known connections, newly connected or disconnected modules can be detected. Newly-connected modules can be interrogated for their full datasheet, while disconnected modules can be removed from memory and removed from future calculations.

An important note, however, is that hardware changes can be missed if devices are quickly swapped on the same socket. For example, if the battery module on socket 2 is swapped for another device, and this happens within the period between scans, the microcontroller will detect a device still present in that socket and not realize that the module has been exchanged. 


\section{Energy-awareness}

The EEDS obtained from each module is used by the embedded software on the microcontroller to assess the overall energy status of the system. For example, with the system interconnection shown in Fig. 4, the amount of energy stored in the supercapacitor is estimated by monitoring its voltage. The energy remaining in the battery is estimated using a $200 \Omega$ impulse load, and compared against the discharge curve provided in the EEDS for the cell.

The status of the mains module is ascertained simply by querying a digital output from the module. The photovoltaic module's nominal power is determined by forcing the photovoltaic cell to be disconnected from its load, and analyzing its open-circuit voltage. As described earlier, its nominal power can then be estimated from the cell parameters given in the EEDS for the module.

The microcontroller classifies the energy status of the node as a discrete power priority level. These are shown in Table V. These effectively provide an input to network algorithms mentioned earlier in this paper. They permit applications to make decisions about activity levels without those applications having to have a detailed knowledge of the details of the energy subsystem on the sensor node. The percentage values given in the table are the default values used in the demonstrator - these can be modified as required.

\section{E. Testing}

The system has been tested with the configuration shown in Fig. 4. The first test performed involved starting with an empty supercapacitor and allowing it to charge from the mains module. The result of this test is shown in Fig. 5. In this test, Channel 1 is the raw voltage on the multiplexer module and

TABLE V. POWER PRIORITY LEVELS

\begin{tabular}{|l|c|l|}
\hline Priority & Max \% & Description \\
\hline PP_Mains & - & Operating from mains power \\
\hline PP_5 & - & Plentiful energy \\
\hline PP_4 & 80 & \multirow{3}{*}{ Intermediate energy levels } \\
\hline PP_3 & 60 & \\
\hline PP_2 & 40 & \\
\hline PP_1 & 20 & Very limited energy \\
\hline PP_Empty & 2 & Cannot sustain activity \\
\hline PP_Err & - & Error calculating status/unknown \\
\hline
\end{tabular}

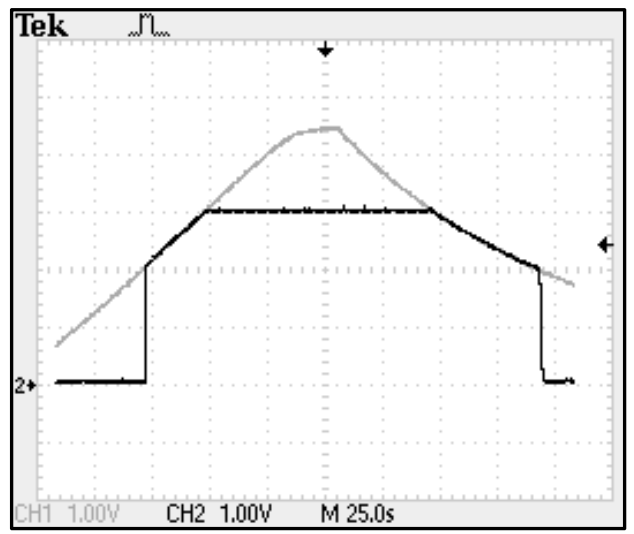

Figure 5 . Node charging with $0.55 \mathrm{~F}$ supercapacitor from mains module, then discharging through $180 \Omega$ resistance.
Channel 2 is the regulated voltage which the microcontroller is connected to. It may be observed that the node reaches the $2.0 \mathrm{~V}$ turn-on voltage after approximately $50 \mathrm{~s}$, and that the regulated voltage continues to rise until it is regulated to $3.0 \mathrm{~V}$. The raw voltage reached a maximum of $4.5 \mathrm{~V}$, before the mains supply was manually turned off and the system was discharged through a $180 \Omega$ resistor. The regulated voltage follows the decay of the raw voltage until it falls below $2.0 \mathrm{~V}$, after which time the microcontroller is disconnected from the supply and the output falls to approximately $0.0 \mathrm{~V}$.

The test system is able to operate autonomously as a sensor node. In the second test, with the Hyperterminal output shown in Fig. 6 being taken from the PC connected to the receiver node, the system was charged up from cold, using the photovoltaic module (which took less than one hour under an indoor light intensity of $900 \mathrm{Lux}$ ) in the configuration shown earlier. The system was then reset, and the initial sweep of the energy subsystem resulted in the first group of transmissions (of the serial number of each of the energy module EPROMs). As the voltage on the supercapacitor is $2.8 \mathrm{~V}$ the system is in PP 3 and wakes up every two seconds to perform a re-scan of its energy hardware.

Approximately 20s after start-up, the vibration module was connected to socket 5 of the multiplexer module. This resulted in an additional transmission showing that a device had been added to socket 5. Approximately $10 \mathrm{~s}$ after this, the photovoltaic module was disconnected from socket 3 and connected to socket 6 , which resulted in two transmissions: firstly to shown that socket 3 was now empty; secondly to show that a device had now been connected to socket 6 .

The third test presented here involved the monitoring of the photovoltaic energy module. The microcontroller was instructed to query the device, and to report the measured value. From this, the open-circuit voltage (and hence nominal power) can be found from the equations given in [13]. With a light intensity measured at 900 Lux, the measurement output from the photovoltaic module was $1.09 \mathrm{~V}$. The unregulated voltage on the multiplexer module was $3.89 \mathrm{~V}$ which led to an estimated open-circuit voltage estimate of $4.54 \mathrm{~V}$. The expected open-circuit voltage at this light intensity was $4.65 \mathrm{~V}$. Therefore the error in the estimated open-circuit voltage is approximately $2 \%$. Given that standard tolerance resistors were used in this module, it is expected that the accuracy of this estimate can be improved substantially.
Initial start-up

$2.852 \mathrm{~V}, 26.0 \mathrm{C}, 1,00000576 \mathrm{c} 739$

$2.852 \mathrm{~V}, 26.0 \mathrm{C}, 2,000005770 \mathrm{~b} 4 \mathrm{~b}$

$2.852 \mathrm{~V}, 26.0 \mathrm{C}, 3,000005770407$

$2.852 \mathrm{~V}, 26.0 \mathrm{C}, 4,00000576 \mathrm{f} 5 \mathrm{~b} 6$

$2.852 \mathrm{~V}, 26.0 \mathrm{C}, 5,000000000000$

$2.852 \mathrm{~V}, 26.0 \mathrm{C}, 6,000000000000$

Connected vibration module to socket 5 . 2.844V,26.0C,5,00000576d4c3

Disconnected photovoltaic module from socket 3 : 2.846V,25.9C,3,000000000000

Reconnected photovoltaic module to socket 6 : $2.846 \mathrm{~V}, 25.3 \mathrm{C}, 6,000005770407$

Figure 6. Annotated Hyperterminal output from second test. 


\section{EVALUATION}

A prototype system has been developed, which facilitates the flexible connection of energy modules as appropriate to the deployment environment and sensing tasks. The scheme allows the sensor node to be powered by a selection of energy resources such as energy harvesting, primary batteries, and a mains power adapter. Energy can also be buffered in rechargeable batteries or supercapacitors. The system autonomously starts up and automatically detects changes in the components of its energy subsystem. The hardware interface has been designed to interface with a range of lowpower microcontrollers.

The developed scheme allows systems to be configured at the time of deployment, gives a great deal of flexibility with regard to the energy hardware, and allows the node to have a more detailed knowledge of its energy subsystem than has previously been possible. The system allows energy modules to be connected to any socket on the multiplexer module, and if necessary multiple modules of the same type can be connected. A major advantage of the plug-and-play functionality is that the system can be installed and configured by a person without an in-depth understanding of the operation of the sensor node.

The main drawbacks are the increased physical size of the system, and the overhead in additional circuitry that delivers the flexibility and energy-aware functionality. Clearly the size and cost of the energy subsystem is higher than those of a longlife battery, but the main advantage of this system is its flexibility with regards to the modules connected to its energy subsystem. It is believed that the enhancements in system reconfigurability and energy-awareness outweigh the other potential drawbacks.

\section{CONCLUSIONS AND FUTURE WORK}

This paper has described a scheme for developing modular plug-and-play power resources for wireless sensor nodes. The complete system, with electronic datasheets and a common hardware interface, delivers a novel plug-and-play capability for the energy subsystems of wireless sensor nodes. The interface allows up to six energy modules to be used to power the sensor node, and the voltages and type of interface used means that the system can be utilized to power sensor nodes based on a range of low-power microcontrollers.

The system has been demonstrated and tested with a range of energy hardware, has been shown to start up and shut down the power supply to a microcontroller dependent on its voltage, and regulate the supply voltage to the microcontroller between $2.0 \mathrm{~V}$ and $3.0 \mathrm{~V}$. The microcontroller software has been demonstrated to detect changes to components of its energy hardware, and it functions as a sensor node detecting and reporting temperature. A system of power priorities have been implemented, which controls the activity of the sensor node dependent on its energy status. The system is now sufficiently developed to interface to a higher-level application, which will allow more comprehensive testing to be carried out, resulting in a truly energy-aware wireless sensor node.

Additional planned future work includes demonstrating the system with an MSP430 microcontroller (in contrast to the CC2430), developing further energy modules (including thermoelectric and wind power) and reducing the overall physical system size. There are also plans to develop a 'lightweight' multiplexer module which would only facilitate the connection of one energy module for simple systems which could function directly from, for example, a long-life primary battery. Ultimately the goal is to develop and evaluate the proposed hardware interface and datasheet structure for a range of microcontrollers and energy devices, thus delivering a truly flexible platform for energy-aware wireless sensor nodes.

\section{REFERENCES}

[1] A True System-on-Chip solution for 2.4 GHz IEEE 802.15.4 / ZigBee ${ }^{\mathrm{TM}}$ [Online], Texas Instruments Inc, Dallas, TX, 2008. Available: http://focus.ti.com/docs/prod/folders/print/cc2430.html

[2] X. Jiang, J. Polastre, and D. Culler, "Perpetual environmentally powered sensor networks," in Proc. IPSN 2005, Los Angeles, CA, 2005, pp. 463468.

[3] R. Torah, P. Glynne-Jones, J. Tudor, T. O’Donnell, S. Roy, and S. Beeby, "Self-powered autonomous wireless sensor node using vibration energy harvesting," in Measurement Scence and Technology, vol. 19, 8 pp., Oct. 2008.

[4] C. Park, and P. Chou, "AmbiMax: autonomous indoor energy harvesting platform for multi-supply wireless sensor nodes," in Proc. SECON 2006, Reston, VA, 2006, pp. 168-177.

[5] R. Morais et al., "Sun, wind and water flow as energy supply for small stationary data acquisition platforms," in Computers and Electronics in Agriculture, vol. 64, no. 2, pp. 120-132, Dec. 2008.

[6] G. V. Merrett, N. R. Harris, B. M. Al-Hashimi, and N. M. White, "Energy managed reporting for wireless sensor networks," in Sensors and Actuators A: Physical, vol. 142, no. 1, pp. 379-389, 2008.

[7] A. S. Weddell, N. R. Harris, N. M. White, "Alternative energy sources for sensor nodes: rationalized design for long-term deployment," in Proc. IEEE $I^{2}$ MTC 2008, Victoria, Canada, pp. 1370-1375, 2008.

[8] IEEE Standard for A Smart Transducer Interface for Sensors and Actuators - Mixed-Mode Communication Protocols and Transducer Electronic Data Sheet (TEDS) Formats, IEEE Standard 1451.4, 2004.

[9] Watlow Sensors [Online], Watlow Ltd., Nottingham, UK, 2009. Available: http://www.watlow.co.uk/products/sensors/

[10] S. Bandari, C. Santiago, H. S. Mohammed, and J. B. Schmalzel, "Component electronic datasheets in ISHM," in Proc. $1^{\text {st }}$ IEEE Sensors Applications Symposium, Houston, TX, 2006, pp. 106-109.

[11] 1-Wire Devices [Online], Maxim Integrated Products, Dallas Semiconductor, Sunnyvale, CA, 2009. Available: www.maximic.com/quick_view2.cfm/qv_pk/2924

[12] MSP430 Ultra-Low Power Microcontrollers [Online], Texas Instruments Inc., Dallas, TX, 2009. Available: http://www.ti.com/msp430

[13] A. Weddell, N. Harris, and N. White, "An efficient indoor photovoltaic power harvesting system for energy-aware wireless sensor nodes," in Proc. Eurosensors XXII, Dresden, Germany, 2008, pp. 1544-1547.

[14] PMG17 Efficacy Data [Online], PMG Perpetuum Ltd., Southampton, UK, 2007. Available: http://perpetuum.co.uk/home.php?page_id=3 